\title{
EXISTENCE AND NONEXISTENCE OF GLOBAL POSITIVE SOLUTIONS TO NONLINEAR DIFFUSION PROBLEMS WITH NONLINEAR ABSORPTION THROUGH THE BOUNDARY
}

\author{
MINGXIN WANG AND YONGHUI WU
}

\begin{abstract}
This paper deals with the existence and nonexistence of global positive solutions to $u_{t}=\Delta \ln (1+u)$ in $\Omega \times(0,+\infty)$,

$$
\frac{\partial \ln (1+u)}{\partial n}=\sqrt{1+u}(\ln (1+u))^{\alpha} \quad \text { on } \partial \Omega \times(0,+\infty),
$$

and $u(x, 0)=u_{0}(x)$ in $\Omega$. Here $\alpha \geq 0$ is a parameter, $\Omega \subset \mathbb{R}^{N}$ is a bounded smooth domain. After pointing out the mistakes in Global behavior of positive solutions to nonlinear diffusion problems with nonlinear absorption through the boundary, SIAM J. Math. Anal. 24 (1993), 317-326, by N. Wolanski, which claims that, for $\Omega=B_{R}$ the ball of $\mathbb{R}^{N}$, the positive solution exists globally if and only if $\alpha \leq 1$, we reconsider the same problem in general bounded domain $\Omega$ and obtain that every positive solution exists globally if and only if $\alpha \leq 1 / 2$.
\end{abstract}

\section{Introduction And Main Results}

In recent years, the existence and nonexistence of global positive solutions to the following nonlinear diffusion equations with nonlinear absorption through the boundary

$$
\begin{cases}u_{t}=\Delta \phi(u) & \text { in } \Omega \times(0, T), \\ \frac{\partial \phi(u)}{\partial n}=f(u) & \text { on } \partial \Omega \times(0, T), \\ u(x, 0)=u_{0}(x) & \text { in } \Omega,\end{cases}
$$

has attracted many authors, where $\Omega \subset \mathbb{R}^{N}(N \geq 1)$ is a bounded domain with smooth boundary $\partial \Omega, n$ is outward normal vector. $\phi, f, \phi^{\prime}$ and $f^{\prime}$ are positive functions for $u>0, \phi$ and $f$ tend to infinity as $u$ goes to infinity. $u_{0} \in L^{\infty}(\Omega)$ is a positive function.

Equation (1.1) includes many well-known equations. For example, $\phi(u)=u^{m}$, (1.1) becomes the famous heat conduction equation if $m=1$, the fast diffusion equation if $m<1$, and the slow diffusion equation if $m>1$. And many applications have been found in physics and chemical, biological and engineering problems, see [2-4]. While the boundary condition in (1.1) can be explained physically as a nonlinear radiation law and here is an absorption law, see [5]. For a proof of local existence and uniqueness of weak solutions when $u_{0}$ is allowed to vanish somewhere

Received by the editors July 13, 1994.

1991 Mathematics Subject Classification. Primary 35K55, 35K60, 35B35.

Key words and phrases. Global solutions, blow up, nonlinear diffusion and absorption, upper and lower solutions.

The first author's work was supported by The National Natural Science Foundation of China. 
and $\phi^{\prime}(0)=0$, we refer to [6], and blow up results for some $u_{0}$ by concavity methods in the one-dimensional case can also be found in [6].

If $u_{0}(x)$ is a positive smooth function and satisfies the compatibility condition $\frac{\partial \phi\left(u_{0}\right)}{\partial n}=f\left(u_{0}\right)$ on $\partial \Omega$, the existence and nonexistence of global solutions to problem (1.1) have been studied by many authors for some special cases. In the case $\phi(u)=$ $u$, i.e. the heat equation, Levine and Payne first studied (1.1) in [5] for $f(u)=$ $|u|^{1+\epsilon} h(u)$ with $\epsilon>0$ and $h(u)$ an increasing positive function. They obtained that the solution may blow up in finite time for some initial data by the concavity methods. Later, Walter dealt with the general nonlinearity $f(u)$ in [7] by use of the comparison principle, and obtained that if $f(u)$ and $f^{\prime}(u)$ are continuous positive and increasing functions, then every solution exists globally if and only if $\int^{+\infty} d s / f(s) f^{\prime}(s)=+\infty$. For the special case $\Omega=B_{R}$, the ball of $\mathbb{R}^{N}$, it was also proved in [8] that every solution blows up in finite time if $\int^{+\infty} d s / f(s)<+\infty$.

For the general nonlinear diffusion case, the situation is more complicated. Under the hypotheses that $\phi^{\prime}(u)$ and $f(u) / \phi^{\prime}(u)$ are positive nondecreasing $C^{1}$ functions for $u>0$, we have proved in [9] that, by use of the upper and lower solution methods, every solution exists globally if and only if $\int^{+\infty} \phi^{\prime}(s) d s / f(s)=+\infty$ and $\int^{+\infty} \phi^{\prime}(s) d s / f(s)\left(\phi^{\prime}(s)+f^{\prime}(s)\right)=+\infty$. However, for the case when $\phi^{\prime}(u)$ is decreasing, the above theorem does not work. For example, when $\phi(u)=u^{m}$ and $f(u)=u^{q}$ with $0<m<1$, [9] cannot deal with it. In this case, if $\Omega=(0,1)$ Filo utilized the integral estimate and the comparison principle to obtain in [10] that if $2 q>m+1$ then every solution blows up in finite time; if $2 q<m+1$ then every solution exists globally. Wu complemented the case $2 q=m+1$ in [11] and obtained that every solution also exists globally. Wang generalized the results of [10], [11] in [12] to general bounded domain of $\mathbb{R}^{N}(N \geq 1)$ and positive bounded initial datum $u_{0}(x)$.

Recently, Wolanski also generalized the above results in [1] to the case $\Omega=B_{R}$, the ball of $\mathbb{R}^{N}$, and the general nonlinearities by use of the maximal principle and integral estimations. For the special nonlinearity $\phi(u)=\ln (1+u)$ and $f(u)=$ $\sqrt{1+u}(\ln (1+u))^{\alpha}$, Wolanski asserted that if $\alpha \leq 1$ then every solution exists globally; if $\alpha>1$ then every solution blows up in finite time. However, we have to point out that this result and Theorem 2.2 of [1] are false, and in the proofs he misused the maximal principle.

In the present paper, we focus our attention on the following problem in general bounded domain $\Omega \subset \mathbb{R}^{N}$ with smooth boundary $\partial \Omega$,

$$
\begin{cases}u_{t}=\Delta \ln (1+u) & \text { in } \Omega \times(0, T), \\ \frac{\partial \ln (1+u)}{\partial n}=\sqrt{1+u}(\ln (1+u))^{\alpha} & \text { on } \partial \Omega \times(0, T), \\ u(x, 0)=u_{0}(x) & \text { in } \Omega,\end{cases}
$$

where $\alpha \geq 0$ is a parameter, $u_{0} \in L^{\infty}(\Omega)$ and $u_{0}(x) \geq \delta>0, \delta$ may be sufficient small. Let $v=\ln (1+u)$, then (1.2) is equivalent to

$$
\begin{cases}\left(e^{v}\right)_{t}=\Delta v & \text { in } \Omega \times(0, T), \\ \frac{\partial v}{\partial n}=v^{\alpha} e^{v / 2} & \text { on } \partial \Omega \times(0, T), \\ v(x, 0)=v_{0}(x) \geq \ln (1+\delta) \triangleq \sigma>0 & \text { in } \Omega .\end{cases}
$$

Our main results read as follows:

Theorem 1. Every solution of (1.3) exists globally if and only if $\alpha \leq 1 / 2$. 
Theorem 2. Assume that $\alpha>1 / 2$ and $\Omega=(-1,1), v_{0}(x)$ satisfies

$(H) v_{0}(x) \in C^{2+\gamma}(\bar{\Omega})$ for some $0<\gamma<1, v_{0}(x)$ satisfies the compatibility conditions at $x= \pm 1, v_{0}(x)=v_{0}(-x)>0$ for $0 \leq x \leq 1, v_{0 x}(x)>0, v_{0 x x}(x)>0$ for $0<x \leq 1$ and $v_{0}^{2}(0)>4 \alpha(\alpha-1)$.

Then the solution $v(x, t)$ of (1.3) satisfies

$$
\begin{gathered}
\lim _{t \rightarrow T_{\max }^{-}} v(1, t)\left(T_{\max }-t\right)^{1 /(2 \alpha-1)} \leq\left(\frac{2}{2 \alpha-1}\right)^{1 /(2 \alpha-1)}, \\
v\left(x, T_{\max }\right)=O(-\ln (1-|x|))
\end{gathered}
$$

as $x$ close to \pm 1 .

If in addition $v_{0}(x) \in C^{3}(\bar{\Omega})$ and satisfies $v_{0 x x x}(x) \geq v_{0 x x}(x) v_{0 x}(x)$ on $[0,1]$, then

$$
\lim _{t \rightarrow T_{\max }} v(1, t)\left(T_{\max }-t\right)^{1 /(2 \alpha-1)}=O(1),
$$

where $T_{\max }<+\infty$ is the blow up time of $v(x, t)$. (1.5) implies that the blow up points are only $x= \pm 1$.

Example. Let $y(x)$ be the solution of

$$
\left\{\begin{array}{l}
y^{\prime}(x)=y^{\alpha}(x) \exp (y(x) / 2)-C(1-x), \quad 0<x \leq 1, \\
y(0)=y_{0},
\end{array}\right.
$$

where $y_{0} \geq \max \{1,2 \sqrt{\alpha|\alpha-1|}\}$ such that $\alpha y_{0}^{\alpha-1} \exp \left(y_{0} / 2\right) \geq 1, C=y_{0}^{\alpha} \exp \left(y_{0} / 2\right)$. It can be proved that $y^{\prime}(x)>0, y^{\prime \prime}(x)>0$ for $0<x \leq 1, y^{\prime}(0)=0$ and $y^{\prime \prime \prime}(x) \geq$ $y^{\prime \prime}(x) y^{\prime}(x)$ on $[0,1]$. Take

$$
v_{0}(x)= \begin{cases}y(x), & 0 \leq x \leq 1 \\ y(-x), & -1 \leq x \leq 0\end{cases}
$$

then $v_{0}(x)$ satisfies $(H)$, and $v_{0}(x) \in C^{3}(\bar{\Omega}), v_{0 x x x}(x) \geq v_{0 x x}(x) v_{0 x}(x)$ on $[0,1]$.

For other papers dealing with the balance between reaction and diffusion or convection, or two reactions of opposite signs, we refer to [13], [14] and the references therein.

The paper is organized as follows. In $\S 2$ we state some results on the local existence and the comparison principle. In $\S 3$ we prove the global existence for $\alpha \leq 1 / 2$ and in $\S 4$ the blow up result for $\alpha>1 / 2$. In $\S 5$ we give the proof of Theorem 2. We will in conclusion raise two remarks on the general problem (1.1).

\section{Local Solutions and Comparison Principle}

Definition. A function $u(x, t)$, defined everywhere on $\bar{\Omega} \times(0, T)$ and almost everywhere on $\bar{\Omega}$, is called a lower (upper) solution of $(1.2)$ on $Q_{T}=\Omega \times(0, T)$ if the following all hold.

(i) $u \in L^{\infty}\left(Q_{\tau}\right)$ for any $0<\tau<T$,

(ii) $u(x, 0) \leq(\geq) u_{0}(x)$ on $\bar{\Omega}$, 
(iii) For every $t \in[0, T)$ and $\psi \in C\left(\bar{Q}_{T}\right) \cap C^{2,1}(\bar{\Omega} \times(0, T)) \cap V\left(Q_{T}\right)$, the following holds.

$$
\begin{aligned}
\int_{\Omega} u(x, t) \psi(x, t) d x \leq & (\geq) \int_{\Omega} u_{0}(x) \psi(x, 0) d x \\
& +\int_{0}^{t} \int_{\Omega}\left[u \psi_{s}+\ln (1+u) \Delta \psi\right] d x d s \\
& +\int_{0}^{t} \int_{\partial \Omega}\left[\sqrt{1+u}(\ln (1+u))^{\alpha} \psi-\ln (1+u) \frac{\partial \psi}{\partial n}\right] d \sigma d s
\end{aligned}
$$

where $V\left(Q_{T}\right)=\left\{\psi\left|\psi_{t},\right| \nabla \psi \mid, \Delta \psi \in L^{2}\left(Q_{T}\right)\right.$ and $\left.\psi \geq 0\right\}$. A function $u(x, t)$ is called a solution of (1.2) on $Q_{T}$ if it is both a lower solution and a upper solution of (1.2) on $Q_{T}$.

By the Theorems 2.1, 2.2 and 2.3 of [6] we have

Proposition 2.1. (i) There exist $T>0$ and a unique noncontinuable solution $u(x, t)$ of $(1.2)$ on $Q_{T}$ satisfying $u(x, t) \geq \delta$.

Moreover, if $T<+\infty$ then

$$
\lim _{t \rightarrow T^{-}}\|u(\cdot, t)\|_{\infty}=+\infty
$$

(ii) Suppose that $\bar{u}(x, t)$ is a upper solution of (1.2) on $Q_{T}$ with initial datum $\bar{u}_{0}(x), \underline{u}(x, t)$ is a lower solution of $(1.2)$ on $Q_{T}$ with initial datum $\underline{u}_{0}(x)$, and $\bar{u}_{0}(x), \underline{u}_{0}(x) \in L^{\infty}(\Omega)$ are positive functions. If $\bar{u}_{0}(x) \geq \underline{u}_{0}(x) \geq \delta$ for some small $\delta>0$, then $\bar{u}(x, t) \geq \underline{u}(x, t)$ in $Q_{T}$.

Using Proposition 2.1 we have

Proposition 2.2. (i) There exist $T>0$ and a unique noncontinuable solution $v(x, t)$ of (1.3) on $Q_{T}$ satisfying $v(x, t) \geq \sigma$.

Moreover, if $T<+\infty$ then

$$
\lim _{t \rightarrow T^{-}}\|v(\cdot, t)\|_{\infty}=+\infty
$$

(ii) Suppose that $\bar{v}(x, t)(\underline{v}(x, t))$ is a classical upper (lower) solution of (1.3), i.e. $\bar{v}(x, t)(\underline{v}(x, t)) \in C^{2,1}(\Omega \times(0, T)) \cap C^{1,0}(\bar{\Omega} \times[0, T))$ and satisfies

$$
\begin{cases}\left(e^{\bar{v}}\right)_{t} \geq \Delta \bar{v} & \left(\left(e^{\underline{v}}\right)_{t} \leq \Delta \underline{v}\right) \quad \text { in } \quad \Omega \times(0, T), \\ \frac{\partial \bar{v}}{\partial n} \geq \bar{v}^{\alpha} e^{\bar{v} / 2} & \left(\frac{\partial \underline{v}}{\partial n} \leq \underline{v}^{\alpha} e^{\underline{v} / 2}\right) \quad \text { on } \quad \partial \Omega \times(0, T), \\ \bar{v}(x, 0) \geq v_{0}(x) & \left(\underline{v}(x, 0) \leq v_{0}(x)\right) \quad \text { in } \quad \Omega .\end{cases}
$$

Then the solution $v(x, t)$ of (1.3) satisfies $v(x, t) \leq \bar{v}(x, t) \quad(v(x, t) \geq \underline{v}(x, t)$ provided that $\underline{v}(x, 0) \geq \sigma$ for some small $\sigma>0)$ in $Q_{T}$.

\section{Proof of Theorem 1 -Global Existence}

In this section, we will prove the first part of Theorem 1 -global existence, i.e. to prove that the solution $v(x, t)$ of (1.3) exists globally if $\alpha \leq 1 / 2$. The basic idea is to construct meticulous upper solutions and by use of the continuation of solutions method. First, we give two lemmas. 
Lemma 3.1. Let $m>0$ and define

$$
s(m)=\int_{m}^{+\infty} \frac{d y}{y^{\alpha} \exp (y / 2)},
$$

then there exists $m^{*} \gg 1$ such that

$$
s(m)>\frac{3}{2} m^{-\alpha} e^{-m / 2} \quad \text { for } m \geq m^{*} .
$$

Proof. Direct computation gives

$$
\lim _{m \rightarrow+\infty} \frac{s(m)}{m^{-\alpha} e^{-m / 2}}=2 .
$$

Hence (3.1) holds.

Lemma 3.2. There exists $m^{*} \gg 1$ such that

$$
\begin{aligned}
& \int_{m}^{m+1} \frac{d y}{y^{\alpha} \exp (y / 2)}<m^{-\alpha} e^{-m / 2} \quad \text { for } m \geq m^{*}, \\
& \int_{m}^{m+6 \ln 2} \frac{d y}{y^{\alpha} \exp (y / 2)}>\frac{3}{2} m^{-\alpha} e^{-m / 2} \quad \text { for } m \geq m^{*} .
\end{aligned}
$$

Proof. Direct computations yield

$$
\begin{gathered}
\lim _{m \rightarrow+\infty} m^{\alpha} e^{m / 2} \int_{m}^{m+1} \frac{d y}{y^{\alpha} \exp (y / 2)}=2\left(1-e^{-1 / 2}\right)<1, \\
\lim _{m \rightarrow+\infty} m^{\alpha} e^{m / 2} \int_{m}^{m+6 \ln 2} \frac{d y}{y^{\alpha} \exp (y / 2)}=2(1-\exp (-3 \ln 2))>\frac{3}{2} .
\end{gathered}
$$

Thus (3.2), (3.3) hold.

We are now in the position to prove the global existence. Assume $\alpha \leq 1 / 2$. Choose $m^{*} \gg 1$, such that (3.1)-(3.3) hold. Let $m \geq m^{*}, m \geq \max _{\bar{\Omega}} v_{0}(x)$, and $\varphi(s)$ be the solution of the following ordinary differential equation

$$
\left\{\begin{array}{l}
\varphi^{\prime}(s)=\varphi^{\alpha}(s) \exp (\varphi(s) / 2), \quad s>0 \\
\varphi(0)=m
\end{array}\right.
$$

Then we have

$$
\int_{m}^{\varphi(s)} \frac{d y}{y^{\alpha} \exp (y / 2)}=s .
$$

It is obvious that there exists $s(m): 0<s(m)<+\infty$ such that $\lim _{s \rightarrow s(m)} \varphi(s)=$ $+\infty$, and $\varphi(s)$ is well defined in $[0, s(m))$. From (3.5) we have

$$
s(m)=\int_{m}^{+\infty} \frac{d y}{y^{\alpha} \exp (y / 2)},
$$

and $s(m)$ satisfies (3.1). Denote $h(x)$ the eigenfunction corresponding to the first eigenvalue $\lambda_{1}$ of $-\Delta$ with Dirichlet boundary condition, i.e.

$$
\begin{cases}-\Delta h=\lambda_{1} h, & x \in \Omega \\ h(x)=0, & x \in \partial \Omega .\end{cases}
$$


Then $\lambda_{1}>0, h(x)>0$ in $\Omega$ and $\frac{\partial h}{\partial n}<0$ on $\partial \Omega$. We may consider $\max _{\bar{\Omega}} h(x) \leq 1 / 2$, and let $\max _{\bar{\Omega}}|\nabla h(x)|=c_{1}$ and $\min _{\partial \Omega}\left|\frac{\partial h}{\partial n}\right|=c_{2}$, then $c_{2}>0$. Take $l=1 / c_{2}, K=$ $3 l^{2} c_{1}^{2}+\lambda_{1} l$ and $g(t)$ be the solution of the following ordinary differential equation

$$
\left\{\begin{array}{l}
g^{\prime}(t)=K \varphi^{\alpha}(g(t)) \exp (-\varphi(g(t)) / 2), \quad t>0 \\
g(0)=0
\end{array}\right.
$$

Using (3.4) we have

$$
K t=\int_{m}^{\varphi(g(t))} \frac{d y}{y^{2 \alpha}} .
$$

Since $2 \alpha \leq 1$, we know that $g(t)$ is well defined on $[0,+\infty)$ and

$$
\lim _{t \rightarrow+\infty} g(t)=s(m)>\frac{3}{2} m^{-\alpha} e^{-m / 2} .
$$

Let $\epsilon=\epsilon(m)=\frac{1}{2} m^{-\alpha} e^{-m / 2}$ and

$$
\bar{v}(x, t)=\varphi\left(g(t)+\epsilon(1-h(x))^{l / \epsilon}\right) .
$$

Since $\epsilon \rightarrow 0$ as $m \rightarrow+\infty$, we can consider $l / \epsilon>2$. Using

$$
g(t)+\epsilon(1-h(x))^{l / \epsilon} \leq g(t)+\epsilon=g(t)+\frac{1}{2} m^{-\alpha} e^{-m / 2},
$$

we see that there exists $T_{1}: 0<T_{1}<+\infty$ such that

$$
g\left(T_{1}\right)+\frac{1}{2} m^{-\alpha} e^{-m / 2}=\frac{3}{2} m^{-\alpha} e^{-m / 2}<s(m),
$$

i.e. $g\left(T_{1}\right)=m^{-\alpha} \exp (-m / 2)$. From (3.10) we have that $\bar{v}(x, t)$ is well defined on $\bar{\Omega} \times\left[0, T_{1}\right]$ and $\bar{v}(x, t) \in C^{2,1}\left(\Omega \times\left(0, T_{1}\right]\right) \cap C^{1,0}\left(\bar{\Omega} \times\left[0, T_{1}\right]\right)$. Using (3.8) it is obtained that

$$
K T_{1}=\int_{m}^{\varphi\left(m^{-\alpha} e^{-m / 2}\right)} \frac{d y}{y^{2 \alpha}} .
$$

Now, we verify that $\bar{v}(x, t)$ is a upper solution of $(1.3)$ on $\bar{\Omega} \times\left[0, T_{1}\right]$. Denote $\tau=g(t)+\epsilon(1-h(x))^{l / \epsilon}$, then we have (remembering $\varphi^{\prime}(\tau)>0$ )

$$
\begin{aligned}
\nabla \bar{v} & =l \varphi^{\prime}(\tau)(1-h(x))^{(l-\epsilon) / \epsilon}(-\nabla h), \\
\triangle \bar{v} & =l^{2} \varphi^{\prime \prime}(\tau)(1-h(x))^{2(l-\epsilon) / \epsilon}|\nabla h|^{2}+l \frac{l-\epsilon}{\epsilon} \varphi^{\prime}(\tau)(1-h(x))^{(l-2 \epsilon) / \epsilon}|\nabla h|^{2} \\
& +l \varphi^{\prime}(\tau)(1-h(x))^{(l-\epsilon) / \epsilon}(-\triangle h) \\
& =l^{2}\left(\alpha \varphi^{\alpha-1}(\tau)+(1 / 2) \varphi^{\alpha}(\tau)\right) \exp (\varphi(\tau) / 2)|\nabla h|^{2}(1-h(x))^{2(l-\epsilon) / \epsilon} \varphi^{\prime}(\tau) \\
& +l \frac{l-\epsilon}{\epsilon}(1-h(x))^{(l-2 \epsilon) / \epsilon}|\nabla h|^{2} \varphi^{\prime}(\tau)+\lambda_{1} l h(x)(1-h(x))^{(l-\epsilon) / \epsilon} \varphi^{\prime}(\tau) \\
& \leq\left[l^{2} c_{1}^{2}\left(\alpha \varphi^{\alpha-1}(\tau)+(1 / 2) \varphi^{\alpha}(\tau)\right) \exp (\varphi(\tau) / 2)+l^{2} c_{1}^{2} / \epsilon+\lambda_{1} l\right] \varphi^{\prime}(\tau) .
\end{aligned}
$$

Since $\varphi(\tau) \geq \varphi(0)=m \gg 1$, we have $\varphi^{\alpha-1}(\tau) \leq \varphi^{\alpha}(\tau)$. Using $\alpha \leq 1 / 2$, it follows that

$$
\begin{aligned}
\triangle \bar{v} & \leq\left[l^{2} c_{1}^{2} \varphi^{\alpha}(\tau) \exp (\varphi(\tau) / 2)+l^{2} c_{1}^{2} / \epsilon+\lambda_{1} l\right] \varphi^{\prime}(\tau) \\
& \leq\left[l^{2} c_{1}^{2}+\left(\left(l^{2} c_{1}^{2} / \epsilon\right)+\lambda_{1} l\right) \varphi^{-\alpha}(\tau) \exp (-\varphi(\tau) / 2)\right] \varphi^{\alpha}(\tau) \exp (\varphi(\tau) / 2) \varphi^{\prime}(\tau) .
\end{aligned}
$$


From $\varphi(\tau) \geq m \gg 1$, it follows that

$$
\begin{aligned}
& \varphi^{-\alpha}(\tau) \exp (-\varphi(\tau) / 2) \leq m^{-\alpha} \exp (-m / 2)=2 \varepsilon \\
& \varphi^{-\alpha}(\tau) \exp (-\varphi(\tau) / 2) \leq 1
\end{aligned}
$$

Thus we have

$$
\begin{aligned}
\triangle \bar{v} & \leq\left(3 l^{2} c_{1}^{2}+\lambda_{1} l\right) \varphi^{\alpha}(\tau) \exp (\varphi(\tau) / 2) \varphi^{\prime}(\tau) \\
& =K \varphi^{\alpha}(\tau) \exp (\varphi(\tau) / 2) \varphi^{\prime}(\tau) .
\end{aligned}
$$

On the other hand

$$
\left(e^{\bar{v}}\right)_{t}=e^{\varphi(\tau)} \varphi^{\prime}(\tau) g^{\prime}(t)
$$

Because $\varphi^{\prime}(\tau)>0$, it follows that if

$$
g^{\prime}(t) \geq K \varphi^{\alpha}(\tau) \exp (-\varphi(\tau) / 2)
$$

then we have

$$
\left(e^{\bar{v}}\right)_{t} \geq \triangle \bar{v} \quad \text { in } \Omega \times\left(0, T_{1}\right] .
$$

Let $f(y)=y^{\alpha} \exp (-y / 2), y>0$, then $f^{\prime}(y)<0$ if and only if $y>2 \alpha$. Since $\tau \geq g(t) \geq 0$, we have $\varphi(\tau) \geq \varphi(g(t)) \geq \varphi(0)=m \geq m^{*}>2 \alpha$. Therefore

$$
K \varphi^{\alpha}(\tau) \exp (-\varphi(\tau) / 2) \leq K \varphi^{\alpha}(g(t)) \exp (-\varphi(g(t)) / 2)=g^{\prime}(t),
$$

i.e. (3.13) holds, and in turn (3.14) holds.

For $x \in \partial \Omega$ and $0 \leq t \leq T_{1}$,

$$
\begin{aligned}
\frac{\partial \bar{v}}{\partial n} & =\varphi^{\prime}(\tau) l(1-h(x))^{(l-\epsilon) / \epsilon}\left(-\frac{\partial h}{\partial n}\right) \\
& =\varphi^{\prime}(\tau) l\left(-\frac{\partial h}{\partial n}\right) \\
& \geq \varphi^{\alpha}(\tau) \exp (\varphi(\tau) / 2) \\
& =\bar{v}^{\alpha} \exp (\bar{v} / 2) . \\
\bar{v}(x, 0)=\varphi(\epsilon(1 & \left.-h(x))^{l / \epsilon}\right)>\varphi(0)=m \geq v_{0}(x), \quad x \in \bar{\Omega} .
\end{aligned}
$$

By Proposition 2.2 we get that the solution $v(x, t)$ of $(1.3)$ satisfies

$$
v(x, t) \leq \bar{v}(x, t), \quad(x, t) \in \bar{\Omega} \times\left[0, T_{1}\right] .
$$

Consequently, $v(x, t)$ exists on $\bar{\Omega} \times\left[0, T_{1}\right]$ and

$$
\begin{aligned}
\max _{\bar{\Omega} \times\left[0, T_{1}\right]} v(x, t) & \leq \max _{\bar{\Omega} \times\left[0, T_{1}\right]} \bar{v}(x, t) \\
& =\varphi\left(g\left(T_{1}\right)+\epsilon\right) \\
& =\varphi\left(\frac{3}{2} m^{-\alpha} e^{-m / 2}\right) .
\end{aligned}
$$

Now, we consider the following problems

$$
\begin{cases}\left(e^{v}\right)_{t}=\Delta v, & (x, t) \in \Omega \times\left(T_{i-1}, T\right), \\ \frac{\partial v}{\partial n}=v^{\alpha} e^{v / 2}, & (x, t) \in \partial \Omega \times\left(T_{i-1}, T\right), \\ \left.v(x, t)\right|_{t=T_{i-1}}=v\left(x, T_{i-1}\right), & x \in \Omega\end{cases}
$$

where $i=1,2, \cdots$.

Denote $m_{1}=m, \varphi_{1}(s)=\varphi(s), g_{1}(t)=g(t), T_{0}=0, \epsilon_{1}=\epsilon(m)=\epsilon\left(m_{1}\right)=$ $\frac{1}{2} m_{1}^{-\alpha} \exp \left(-m_{1} / 2\right)$ and $\bar{v}_{1}(x, t)=\bar{v}(x, t)$. Let

$$
m_{i+1}=\varphi_{i}\left(\frac{3}{2} m_{i}^{-\alpha} e^{-m_{i} / 2}\right),
$$




$$
\begin{gathered}
\epsilon_{i}=\frac{1}{2} m_{i}^{-\alpha} e^{-m_{i} / 2}, \\
\bar{v}_{i}(x, t)=\varphi_{i}\left(g_{i}(t)+\epsilon_{i}(1-h(x))^{l / \epsilon_{i}}\right),
\end{gathered}
$$

where $\varphi_{i}(s)$ and $g_{i}(t)$ are the solutions of the following ordinary differential equations

$$
\left\{\begin{array}{l}
\varphi_{i}^{\prime}(s)=\varphi_{i}^{\alpha}(s) \exp \left(\varphi_{i}(s) / 2\right), \quad s>0 \\
\varphi_{i}(0)=m_{i}
\end{array}\right.
$$

and

$$
\left\{\begin{array}{l}
g_{i}^{\prime}(t)=K \varphi_{i}^{\alpha}\left(g_{i}(t)\right) \exp \left(-\varphi_{i}\left(g_{i}(t)\right) / 2\right), \quad t>T_{i-1} \\
g_{i}\left(T_{i-1}\right)=0
\end{array}\right.
$$

respectively, $i=1,2, \cdots$.

Remembering (3.15), repeating the above procedure of $i=1$ we get that $\bar{v}_{2}(x, t)$ $=\varphi_{2}\left(g_{2}(t)+\epsilon_{2}(1-h(x))^{l / \epsilon_{2}}\right)$ is upper solution of $\left(3.16_{2}\right)$ on $\bar{\Omega} \times\left[T_{1}, T_{2}\right]$ and $T_{2}$ satisfies

$$
K\left(T_{2}-T_{1}\right)=\int_{m_{2}}^{\varphi_{2}\left(m_{2}^{-\alpha} e^{-m_{2} / 2}\right)} \frac{d y}{y^{2 \alpha}} .
$$

Therefore $v(x, t) \leq \bar{v}_{2}(x, t)$ on $\bar{\Omega} \times\left[T_{1}, T_{2}\right]$, and hence

$$
\begin{aligned}
\max _{\bar{\Omega} \times\left[T_{1}, T_{2}\right]} v(x, t) & \leq \max _{\bar{\Omega} \times\left[T_{1}, T_{2}\right]} \bar{v}_{2}(x, t) \\
& =\varphi_{2}\left(g_{2}\left(T_{2}\right)+\epsilon_{2}\right) \\
& =\varphi_{2}\left(\frac{3}{2} m_{2}^{-\alpha} e^{-m_{2} / 2}\right)=m_{3} .
\end{aligned}
$$

Continuing the above procedures and by the inductive method we have that

$$
v(x, t) \leq \bar{v}_{i}(x, t) \quad \text { on } \bar{\Omega} \times\left[T_{i-1}, T_{i}\right], \quad i=1,2, \cdots,
$$

and $T_{i}$ satisfies

$$
K\left(T_{i}-T_{i-1}\right)=\int_{m_{i}}^{\varphi_{i}\left(m_{i}^{-\alpha} e^{-m_{i} / 2}\right)} \frac{d y}{y^{2 \alpha}}, \quad i=1,2, \cdots .
$$

Thus $v(x, t)$ exists on $\bar{\Omega} \times\left[0, T_{\infty}\right)$, and

$$
\begin{aligned}
K T_{\infty} & =K \lim _{i \rightarrow+\infty} T_{i}=K \sum_{i=1}^{\infty}\left(T_{i}-T_{i-1}\right) \\
& =\sum_{i=1}^{\infty} \int_{m_{i}}^{\varphi_{i}\left(m_{i}^{-\alpha} e^{-m_{i} / 2}\right)} \frac{d y}{y^{2 \alpha}} .
\end{aligned}
$$

From (3.17) we have

$$
s=\int_{m_{i}}^{\varphi_{i}(s)} \frac{d y}{y^{\alpha} e^{y / 2}}
$$

and hence

$$
m_{i}^{-\alpha} e^{-m_{i} / 2}=\int_{m_{i}}^{\varphi_{i}\left(m_{i}^{-\alpha} e^{-m_{i} / 2}\right)} \frac{d y}{y^{\alpha} e^{y / 2}}
$$




$$
\begin{aligned}
\frac{3}{2} m_{i}^{-\alpha} e^{-m_{i} / 2} & =\int_{m_{i}}^{\varphi_{i}\left(\frac{3}{2} m_{i}^{-\alpha} e^{-m_{i} / 2}\right)} \frac{d y}{y^{\alpha} e^{y / 2}} \\
& =\int_{m_{i}}^{m_{i+1}} \frac{d y}{y^{\alpha} e^{y / 2}} .
\end{aligned}
$$

Because $\varphi_{i}^{\prime}(s)>0$, it is easy to obtain that $m_{i}>m_{i-1}>\cdots>m_{1}=m \geq m^{*}$. (3.2) and (3.19), (3.3) and (3.20) yield

$$
m_{i}+1<\varphi_{i}\left(m_{i}^{-\alpha} e^{-m_{i} / 2}\right)<m_{i+1}<m_{i}+6 \ln 2 .
$$

By induction we have

$$
m_{1}+i<m_{i+1}<m_{1}+i(6 \ln 2) .
$$

In the following we prove $T_{\infty}=+\infty$. We first consider the case $\alpha=1 / 2$. By (3.18), (3.21) and (3.22), it follows

$$
\begin{aligned}
K T_{\infty} & =\sum_{i=1}^{\infty} \ln \frac{\varphi_{i}\left(m_{i}^{-\alpha} e^{-m_{i} / 2}\right)}{m_{i}} \\
& \geq \sum_{i=2}^{\infty} \ln \frac{m_{i}+1}{m_{i}}=\sum_{i=2}^{\infty} \ln \left(1+\frac{1}{m_{i}}\right) \\
& \geq \sum_{i=1}^{\infty} \ln \left(1+\frac{1}{m_{1}+i(6 \ln 2)}\right) \\
& =+\infty
\end{aligned}
$$

Next, we consider the case $\alpha<1 / 2$. It yields $1-2 \alpha>0$. By (3.18), (3.21) and (3.22), we have

$$
\begin{aligned}
K T_{\infty} & =\sum_{i=1}^{\infty} \frac{1}{1-2 \alpha}\left[\varphi_{i}^{1-2 \alpha}\left(m_{i}^{-\alpha} e^{-m_{i} / 2}\right)-m_{i}^{1-2 \alpha}\right] \\
& \geq \frac{1}{1-2 \alpha} \sum_{i=1}^{\infty}\left[\left(m_{i}+1\right)^{1-2 \alpha}-m_{i}^{1-2 \alpha}\right] .
\end{aligned}
$$

Since

$$
\lim _{y \rightarrow+\infty} \frac{(1+y)^{1-2 \alpha}-y^{1-2 \alpha}}{y^{-2 \alpha}}=1-2 \alpha
$$

and

$$
\sum_{i=1}^{\infty} \frac{1}{m_{i}^{2 \alpha}} \geq \sum_{i=2}^{\infty} \frac{1}{\left(m_{1}+i(6 \ln 2)\right)^{2 \alpha}}=+\infty
$$

by (3.23) it follows that $T_{\infty}=+\infty$. Therefore, $v(x, t)$ exists globally. The proof of global existence is completed. 


\section{Proof of Theorem 1-Blow Up Result}

In this section, we shall prove the second part of Theorem 1-blow up result, i.e. to prove that every solution of (1.3) blows up in finite time if $\alpha>1 / 2$. The main idea is to construct lower solution of (1.3) which blows up in finite time.

Since $v_{0}(x) \geq \sigma>0$, choosing $0<\epsilon<\sigma$ and let $\varphi(s)$ be the solution of the ordinary differential equation

$$
\left\{\begin{array}{l}
\varphi^{\prime}(s)=\varphi^{\alpha}(s) \exp (\varphi(s) / 2), \quad s>0 \\
\varphi(0)=\epsilon
\end{array}\right.
$$

Then $\varphi^{\prime}(s)>0$, and there exists $s_{0}: 0<s_{0}<+\infty$, such that

$$
\lim _{s \rightarrow s_{0}^{-}} \varphi(s)=+\infty \text {. }
$$

Thanks to $0<\epsilon<\sigma$, we have that there exists $s_{1}: 0<s_{1}<s_{0}$ such that

$$
\varphi(s) \leq \sigma \text { for all } 0 \leq s \leq s_{1} .
$$

Let $g(t)$ be the solution of the ordinary differential equation

$$
\left\{\begin{array}{l}
g^{\prime}(t)=c_{3} \varphi^{\alpha}\left(g(t)+c_{4}\right) \exp \left(-\varphi\left(g(t)+c_{4}\right) / 2\right), \quad t>0 \\
g(0)=0
\end{array}\right.
$$

where

$$
\begin{gathered}
c_{3}=\min \left\{\frac{1}{2} N c_{5}^{2}, \frac{1}{2} N c_{5}^{2}(\epsilon / 2 \alpha)^{\alpha}\right\}, \quad c_{4}=2 s_{1} / 3, \\
0<c_{5}<\min \left\{1 / N, s_{1} /(6 N d+3)\right\}, \\
d>0 \text { satisfying }\left|x_{i}\right|<d \text { for any } x=\left(x_{1}, \cdots, x_{N}\right) \in \bar{\Omega} .
\end{gathered}
$$

From (4.2) we have

$$
\int_{\varphi\left(c_{4}\right)}^{\varphi\left(g(t)+c_{4}\right)} \frac{d y}{y^{2 \alpha}}=c_{3} t
$$

Since $\alpha>1 / 2$, we get that there exists $T_{0}: 0<T_{0}<+\infty$ such that

$$
g\left(T_{0}\right)+c_{4}=s_{0} \text {, i.e. } \lim _{t \rightarrow T_{0}^{-}} \varphi\left(g(t)+c_{4}\right)=+\infty .
$$

Let

$$
\underline{v}(x, t)=\varphi\left(g(t)+k+c_{5}(h(x)+A)\right),
$$

where

$$
\begin{aligned}
& A=N d+1, h(x)=x_{1}+\cdots+x_{N}, \\
& k \geq 0 \text { satisfying } k+\max _{\bar{\Omega}}\left\{c_{5}(h(x)+A)\right\}=c_{4} .
\end{aligned}
$$

It is easy to see that $\underline{v}(x, t)$ is well defined on $\bar{\Omega} \times\left[0, T_{0}\right)$. Using (4.3) we have

$$
\lim _{t \rightarrow T_{0}^{-}}\|\underline{v}(\cdot, t)\|_{\infty}=\lim _{t \rightarrow T_{0}^{-}} \varphi\left(g(t)+c_{4}\right)=+\infty .
$$

Denote $\tau=g(t)+k+c_{5}(h(x)+A)$, then we have

$$
\begin{gathered}
\left(e^{\underline{v}}\right)_{t}=e^{\varphi(\tau)} \varphi^{\prime}(\tau) g^{\prime}(t), \\
\nabla \underline{v}=c_{5} \varphi^{\prime}(\tau) \nabla h,
\end{gathered}
$$




$$
\begin{aligned}
\triangle \underline{v} & =c_{5}^{2} \varphi^{\prime \prime}(\tau)|\nabla h|^{2} \\
& =N c_{5}^{2}\left[\alpha \varphi^{\alpha-1}(\tau)+\frac{1}{2} \varphi^{\alpha}(\tau)\right] \exp (\varphi(\tau) / 2) \varphi^{\prime}(\tau) \\
& \geq \frac{1}{2} N c_{5}^{2} \varphi^{\alpha}(\tau) \exp (\varphi(\tau) / 2) \varphi^{\prime}(\tau) .
\end{aligned}
$$

Since $\varphi^{\prime}(\tau)>0$, it is obvious that if

$$
g^{\prime}(t) \leq \frac{1}{2} N c_{5}^{2} \varphi^{\alpha}(\tau) \exp (-\varphi(\tau) / 2)
$$

then we have

$$
\left(e^{\underline{v}}\right)_{t} \leq \triangle \underline{v} \quad \text { in } \Omega \times\left(0, T_{0}\right)
$$

Let $f(y)=y^{\alpha} e^{-y / 2}$ for $y>0$, then we have

$$
f^{\prime}(y)=\left(\alpha y^{\alpha-1}-\frac{1}{2} y^{\alpha}\right) e^{-y / 2} .
$$

For $y_{0}>0, f^{\prime}\left(y_{0}\right)=0$ if and only if $y_{0}=2 \alpha$. It is easy to calculate that $f^{\prime \prime}\left(y_{0}\right)=$ $f^{\prime \prime}(2 \alpha)<0$. Therefore,

$$
\left\{\begin{array}{l}
f^{\prime}(y)<0 \text { for } y>2 \alpha \\
\max _{y \geq 0} f(y) \leq f(2 \alpha)=(2 \alpha)^{\alpha} e^{-\alpha}
\end{array}\right.
$$

For any $(x, t) \in \bar{\Omega} \times\left[0, T_{0}\right)$, if $\varphi(\tau)=\varphi\left(g(t)+k+c_{5}(h(x)+A)\right) \leq 2 \alpha$, then we have

$$
\begin{gathered}
\varphi^{\alpha}(\tau) \exp (-\varphi(\tau) / 2) \geq \varphi^{\alpha}(0) e^{-\alpha}=\epsilon^{\alpha} e^{-\alpha}, \\
\varphi^{\alpha}\left(g(t)+c_{4}\right) \exp \left(-\varphi\left(g(t)+c_{4}\right) / 2\right) \leq f(2 \alpha)=(2 \alpha)^{\alpha} e^{-\alpha} .
\end{gathered}
$$

By the choice of $c_{3}$, from (4.2), (4.8) and (4.9) it follows that (4.5) holds. For any $(x, t) \in \bar{\Omega} \times\left[0, T_{0}\right)$, if $\varphi(\tau)>2 \alpha$, thanks to $g(t)+c_{4} \geq \tau$ and $\varphi^{\prime}(s)>0$, we have $\varphi\left(g(t)+c_{4}\right) \geq \varphi(\tau)>2 \alpha$. And (4.5) follows directly from (4.7) and (4.2), and in turn (4.6) holds.

For $(x, t) \in \partial \Omega \times\left(0, T_{0}\right)$, we have

$$
\begin{gathered}
\frac{\partial \underline{v}}{\partial n}=c_{5} \varphi^{\prime}(\tau) \frac{\partial h}{\partial n} \leq N c_{5} \varphi^{\prime}(\tau) \leq \varphi^{\prime}(\tau)=\underline{v}^{\alpha} \exp (\underline{v} / 2) . \\
\underline{v}(x, 0)=\varphi\left(k+c_{5}(h(x)+A)\right) \leq \varphi\left(s_{1}\right) \leq \sigma \leq v_{0}(x), \quad x \in \bar{\Omega} .
\end{gathered}
$$

By Proposition 2.2 we have that the solution $v(x, t)$ of (1.3) satisfies

$$
v(x, t) \geq \underline{v}(x, t) \text { on } \bar{\Omega} \times\left[0, T_{0}\right) .
$$

From (4.4) it follows that $v(x, t)$ blows up in finite time. The proof of the blow up result is completed. 


\section{Proof of Theorem 2}

Throughout this section we assume that $(H)$ holds. Since $\alpha>\frac{1}{2}$, by Theorem 1 we know that the solution $v(x, t)$ blows up in finite time $T_{\max }$. By the standard method, it is easy to prove that the solution $v(x, t)$ of $(1.3)$ is a radially symmetric positive classical solution, i.e. $v(x, t) \in C^{2+\gamma}\left(\bar{\Omega} \times\left(0, T_{\max }\right)\right) \cap C(\bar{\Omega} \times$ $\left.\left[0, T_{\max }\right)\right), v(x, t)=v(-x, t)>0$ for $0 \leq x \leq 1, v_{x}(0, t)=0$. And $v_{x}(x, t)>$ $0, v_{t}(x, t) \geq 0, \not \equiv 0$ for $0<x \leq 1,0<t<T_{\max }$.

Proof of (1.4). Using assumption $(H)$, it is easy to verify that

$$
v_{0 x}(x) \leq v_{0}^{\alpha}(x) \exp \left(v_{0}(x) / 2\right)+k_{1}(1-x), \quad 0 \leq x \leq 1,
$$

where $k_{1}=c_{1}+c_{2}, c_{1}=\max _{[-1,1]}\left\{\left[v_{0}^{\alpha}(x) / 2+\alpha v_{0}^{\alpha-1}(x)\right] v_{0 x}(x) \exp \left(v_{0}(x) / 2\right)\right\}, c_{2}=$ $\max _{[-1,1]}\left|v_{0 x x}(x)\right|$.

Let $H(x, t)=v_{x}-v^{\alpha} e^{v / 2}-k_{1}(1-x) e^{v}$, then we have, using (5.1),

$$
\begin{cases}e^{v} H_{t}-H_{x x}+v_{x} H_{x} \leq 0 & \text { in }(0,1) \times\left(0, T_{\max }\right), \\ H(0, t)<0, H(1, t)=0 & \text { in }\left(0, T_{\max }\right), \\ H(x, 0) \leq 0 & \text { on }[0,1] .\end{cases}
$$

Therefore, $H(x, t) \leq 0$ on $[0,1] \times\left[0, T_{\max }\right)$. Which yields

$$
0<v_{x}(x, t) \leq v^{\alpha} e^{v / 2}+k_{1}(1-x) e^{v} \quad \text { in }(0,1] \times\left[0, T_{\max }\right) .
$$

Using (5.2) we have, for $0<t<T_{\max }$,

i.e.

$$
\begin{aligned}
v_{x x}(1, t) & =\lim _{x \rightarrow 1^{-}} \frac{v_{x}(1, t)-v_{x}(x, t)}{1-x} \\
& \geq\left(\frac{1}{2} v^{2 \alpha}(1, t)+\alpha v^{2 \alpha-1}(1, t)-k_{1}\right) e^{v(1, t)},
\end{aligned}
$$

$$
v_{t}(1, t) \geq \frac{1}{2} v^{2 \alpha}(1, t)+\alpha v^{2 \alpha-1}(1, t)-k_{1}, \quad 0<t<T_{\max }
$$

Since $v_{x}(x, t)>0$ and $v(x, t)$ blows up in $T_{\max }$, we have $v(1, t) \rightarrow+\infty$ as $t \rightarrow T_{\max }$, hence (5.3) yields

$$
v_{t}(1, t) \geq \frac{1}{2} v^{2 \alpha}(1, t)
$$

when $t$ is close to $T_{\max }$. In view of $2 \alpha>1$ we have

$$
\lim _{t \rightarrow T_{\max }^{-}} v(1, t)\left(T_{\max }-t\right)^{1 /(2 \alpha-1)} \leq\left(\frac{2}{2 \alpha-1}\right)^{1 /(2 \alpha-1)},
$$

i.e. (1.4).

Proof of (1.5). Let $w=(1-x) v_{x}(x, t)-2, w$ satisfy

$$
\left\{\begin{array}{lll}
e^{v} w_{t}-w_{x x}+e^{v} v_{t} w=0 & \text { in }(0,1) \times\left(0, T_{\max }\right), \\
w(0, t)=-2, w(1, t)=-2 & \text { on }\left[0, T_{\max }\right), \\
w(x, 0)=(1-x) v_{0 x}(x)-2 & \text { on }[0,1] .
\end{array}\right.
$$

Therefore

which yields

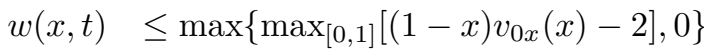

$$
\begin{aligned}
& \leq \max _{[0,1]}(1-x) v_{0 x}(x),
\end{aligned}
$$

$$
(1-x) v_{x}(x, t) \leq 2+\max _{[0,1]}(1-x) v_{0 x}(x) \triangleq k_{2} \quad \text { on }[0,1] \times\left[0, T_{\max }\right) .
$$


Denote $z(x, t)$ by the solution of the following problem:

$$
\left\{\begin{array}{l}
z_{t}-z_{x x}=0,0<x<\frac{1}{2}, t>0 \\
z_{x}(0, t)=0, z_{x}\left(\frac{1}{2}, t\right)=2 k_{2}, t>0 \\
z(x, 0)=v_{0}(x)+\left[2 k_{2}-v_{0 x}\left(\frac{1}{2}\right)\right] x^{2}, 0 \leq x \leq \frac{1}{2} .
\end{array}\right.
$$

Then $z(x, t)$ exists globally. Let

$$
y(x, t)=\left(\max _{[0,1]} v_{0 x x}(x)+4 k_{2}\right) t+v_{0}(x)+\left[2 k_{2}-v_{0 x}\left(\frac{1}{2}\right)\right] x^{2} .
$$

It is easy to verify that $y(x, t)$ is a upper solution of $(5.5)$, hence we have

$$
0<z(x, t) \leq y(x, t) \leq c(1+t), \quad 0 \leq x \leq \frac{1}{2}, \quad t>0 .
$$

Using (5.4) we see that $v(x, t)$ is a lower solution of $(5.5)$ on $\left[0, \frac{1}{2}\right] \times\left[0, T_{\max }\right)$, consequently

$$
v(x, t) \leq z(x, t) \leq c(1+t) \quad \text { on }\left[0, \frac{1}{2}\right] \times\left[0, T_{\max }\right),
$$

which yields

$$
v(0, t) \leq c(1+t), \quad 0 \leq t<T_{\max } .
$$

By means of (5.4) and (5.6) we have

$$
v(x, t) \leq v(0, t)-k_{2} \ln (1-x) \leq c(1+t)-k_{2} \ln (1-x) \quad \text { in }(0,1) \times\left(0, T_{\max }\right) .
$$

This shows that the blow up points are only $x= \pm 1$. On the other hand, using (5.2) we get

$$
e^{-v} v_{x} \leq v^{\alpha} e^{-v / 2}+k_{1}(1-x) .
$$

Integrating this inequality from $x$ to 1 we obtain

$$
e^{-v(x, t)}-e^{-v(1, t)} \leq \int_{x}^{1} v^{\alpha}(s, t) e^{-v(s, t) / 2} d s+k_{1}(1-x)^{2} / 2 .
$$

It is easy to calculate that the right hand side of (5.8) is bounded by

$$
\left((2 \alpha)^{\alpha} e^{-\alpha}+k_{1} / 2\right)(1-x) \text {. }
$$

Let $t \rightarrow T_{\max }$ in (5.8) we have

$$
\exp \left\{-v\left(x, T_{\max }\right)\right\} \leq\left((2 \alpha)^{\alpha} e^{-\alpha}+k_{1} / 2\right)(1-x),
$$

and hence

$$
v\left(x, T_{\max }\right) \geq-c-\ln (1-x) .
$$

This inequality and (5.7) show that (1.5) holds.

Finally, for prowing (1.6), it remains to get a lower bound of blow up rate of $v(x, t)$. To do so, we first prove

Lemma 5.1. If $v_{0}(x) \in C^{3}(\bar{\Omega})$ and $v_{0 x x x}(x) \geq v_{0 x x}(x) \cdot v_{0 x}(x)$ on $[0,1]$, then $v_{x t}(x, t) \geq 0$, i.e. $v_{x}(x, t)$ is nondeereasing in $t$.

Proof. Let $z=v_{x}$, then we have, by (1.3),

$$
\begin{cases}e^{v} z_{t}-z_{x x}+z z_{x}=0 & \text { in }(0,1) \times\left(0, T_{\max }\right), \\ z(0, t)=0, \quad z(1, t)=v^{\alpha}(1, t) e^{v(1, t) / 2} & \text { in } \quad\left(0, T_{\max }\right), \\ z(x, 0)=z_{0}(x)=v_{0 x}(x) \geq 0 & \text { on }[0,1] .\end{cases}
$$


By the assumptions on $v_{0}(x)$, we see that $z_{0}(x)=v_{0 x}(x)$ satisfies the compatibility conditions of $(5.9)$ at $x=0,1$, and $z_{0}(x) z_{0 x}(x) \leq z_{0 x x}(x)$ on $[0,1]$, i.e. $z_{0}(x)$ is a lower solution of (5.9). Using the standard method we have $z_{t} \geq 0$, i.e. $v_{x t}(x, t) \geq 0$ in $(0,1) \times\left(0, T_{\max }\right)$.

Lemma 5.2. Under the assumptions of Lemma 5.1, there exists $T_{1}: 0<T_{1}<$ $T_{\max }$ such that

$$
x v_{t}(x, t) \leq v_{x}(x, t)+\max _{[0,1]}\left(x v_{x x}\left(x, T_{1}\right) e^{-v\left(x, T_{1}\right)}\right) \quad \text { in } \quad(0,1) \times\left[T_{1}, T_{\max }\right) .
$$

Proof. Since $v(1, t) \rightarrow+\infty$ as $t \rightarrow T_{\max }$, there exists $T_{1}: 0<T_{1}<T_{\max }$, such that

$$
\left(\frac{1}{2} v^{\alpha}(1, t)+\alpha v^{\alpha-1}(1, t)\right) \exp (v(1, t) / 2) \leq e^{v(1, t)}-1 \quad \text { for } \quad T_{1} \leq t<T_{\max } .
$$

Denote $k_{3}=\max _{[0,1]}\left(x v_{x x}\left(x, T_{1}\right) e^{-v\left(x, T_{1}\right)}\right)$, and let

$$
w(x, t)=x v_{t}-v_{x}-k_{3}
$$

Then we have, by using Lemma 5.1,

$$
\begin{aligned}
& e^{v} w_{t}-w_{x x}+e^{v} v_{t} w=-2 v_{x t}-k_{3} e^{v} v_{t} \leq 0 \quad \text { in }(0,1) \times\left(T_{1}, T_{\max }\right), \\
& w(0, t)=-k_{3}<0 \quad \text { in }\left(T_{1}, T_{\max }\right) \text {, } \\
& w_{x}(1, t)=\left.\left[1-e^{v}+\left(\alpha v^{\alpha-1}+\frac{1}{2} v^{\alpha}\right) e^{v / 2}\right] e^{-v} v_{x x}\right|_{x=1} \leq 0 \quad \text { in }\left(T_{1}, T_{\max }\right), \\
& w\left(x, T_{1}\right)=x v_{t}\left(x, T_{1}\right)-v_{x}\left(x, T_{1}\right)-k_{3} \\
& =x e^{-v\left(x, T_{1}\right)} v_{x x}\left(x, T_{1}\right)-k_{3}-v_{x}\left(x, T_{1}\right) \\
& \leq 0 \quad \text { on }[0,1] \text {. }
\end{aligned}
$$

Therefore $w(x, t) \leq 0$ on $[0,1] \times\left[T_{1}, T_{\max }\right)$, and in turn (5.10) holds.

Lemma 5.3. Under the assumptions of Lemma 5.1, there exists $k_{4}>0$ which depends only on $v_{0}(x), \alpha$ and $T_{1}$, such that

$$
e^{v} v_{t} \leq v_{x}^{2}+k_{4} v_{x} \quad \text { on } \quad\left[\frac{1}{2}, 1\right] \times\left[T_{1}, T_{\max }\right) .
$$

Proof. By Lemma 5.1 and $v_{x}>0$ in $(0,1]$, we know that there exists $\epsilon>0$ such that

$$
v_{x}(x, t) \geq \epsilon \quad \text { on } \quad\left[\frac{1}{2}, 1\right] \times\left[T_{1}, T_{\max }\right)
$$

Using (5.4) and (5.10) we have, for $T_{1} \leq t<T_{\max }$,

$$
v_{t}\left(\frac{1}{2}, t\right) \leq 2\left(v_{x}\left(\frac{1}{2}, t\right)+k_{3}\right) \leq 2\left(2 k_{2}+k_{3}\right)
$$

which yields, for $T_{1} \leq t<T_{\max }$,

$$
v\left(\frac{1}{2}, t\right) \leq v\left(\frac{1}{2}, T_{1}\right)+\left(4 k_{2}+2 k_{3}\right)\left(T_{\max }-T_{1}\right) \triangleq c_{1} .
$$

Since $v(1, t) \rightarrow+\infty$ as $t \rightarrow T_{\max }$, without loss of generality we may think that $v(1, t)>2 \alpha$ for all $T_{1} \leq t<T_{\max }$. Let

$$
\begin{aligned}
& k_{4}=\frac{1}{\epsilon}\left\{\max _{[0,1]}\left(e^{v\left(x, T_{1}\right)} v_{t}\left(x, T_{1}\right)\right)+2\left(2 k_{2}+k_{3}\right) e^{c_{1}}\right\}, \\
& w(x, t)=v_{x}^{2}+k_{4} v_{x}-e^{v} v_{t},
\end{aligned}
$$


then we have, by (5.12)-(5.14),

$$
\begin{aligned}
e^{v} w_{t}-w_{x x}+2 v_{x} w_{x}-e^{v} v_{t} w=0 & \text { in }\left[\frac{1}{2}, 1\right) \times\left(T_{1}, T_{\max }\right), \\
w\left(x, T_{1}\right)>0 & \text { in }\left[\frac{1}{2}, 1\right], \\
w\left(\frac{1}{2}, t\right)>0 & \text { in }\left[T_{1}, T_{\max }\right), \\
w_{x}(1, t) & =v_{x x}(1, t)\left[v^{\alpha}(1, t) e^{v(1, t) / 2}+k_{4}-\left(\frac{1}{2} v^{\alpha}(1, t)+\alpha v^{\alpha-1}(1, t)\right) e^{v(1, t) / 2}\right] \\
& >v_{x x}(1, t)\left[\frac{1}{2} v^{\alpha}(1, t)-\alpha v^{\alpha-1}(1, t)\right] e^{v(1, t) / 2} \\
& \geq 0 \quad \text { in }\left[T_{1}, T_{\max }\right)
\end{aligned}
$$

since $v(1, t)>2 \alpha$ and $v_{x x}(1, t) \geq 0$. By the maximum principle we have

$$
w(x, t) \geq 0 \quad \text { on } \quad\left[\frac{1}{2}, 1\right) \times\left[T_{1}, T_{\max }\right),
$$

and hence (5.11) holds.

From (5.11) we have, for $T_{1} \leq t<T_{\max }$,

$$
\begin{aligned}
v_{t}(1, t) & \leq e^{-v(1, t)}\left(v_{x}^{2}(1, t)+k_{4} v_{x}(1, t)\right) \\
& =e^{-v(1, t)}\left(v^{2 \alpha}(1, t) e^{v(1, t)}+k_{4} v^{\alpha}(1, t) e^{v(1, t) / 2}\right) \\
& =v^{2 \alpha}(1, t)+k_{4} v^{\alpha}(1, t) e^{-v(1, t) / 2} .
\end{aligned}
$$

By use of $v(1, t) \rightarrow+\infty$ as $t \rightarrow T_{\max }$, we obtain easily that

$$
v(1, t) \geq\left\{(2 \alpha-1)\left[1+k_{4} v^{-\alpha}(1, t) e^{-v(1, t) / 2}\right]\left(T_{\max }-t\right)\right\}^{1 /(1-2 \alpha)},
$$

and therefore

$$
\lim _{t \rightarrow T_{\max }^{-}} v(1, t)\left(T_{\max }-t\right)^{1 /(2 \alpha-1)} \geq\left(\frac{1}{2 \alpha-1}\right)^{1 /(2 \alpha-1)} .
$$

This inequality and (1.4) show that (1.6) holds.

\section{Concluding Remarks}

Remark 1. Considering the general problem

$$
\begin{cases}\left(e^{u}\right)_{t}=\Delta u & \text { in } \Omega \times(0, T), \\ \frac{\partial u}{\partial n}=u^{\alpha} e^{\beta u}(\ln (1+u))^{l} & \text { on } \partial \Omega \times(0, T), \\ u(x, 0)=u_{0}(x) & \text { in } \Omega,\end{cases}
$$

where $\Omega \subset \mathbb{R}^{N}$ is a bounded domain with smooth boundary $\partial \Omega, \alpha, \beta, l \geq 0$, are the parameters. $u_{0} \in L^{\infty}(\Omega)$ and $u_{0}(x) \geq \delta$ for some small $\delta>0$. Similar to the proof of Theorem 1 we can prove the following results.

(I). If $\beta>1 / 2$, then every solution blows up in finite time;

(II). If $\beta<1 / 2$, then every solution exists globally;

(III). If $\beta=1 / 2$, then we have the following three cases:

(i) if $\alpha>1 / 2$, then every solution blows up in finite time;

(ii) if $\alpha<1 / 2$, then every solution exists globally;

(iii) if $\alpha=1 / 2$, then $l>1 / 2$ implies that every solution blows up in finite time; $l \leq 1 / 2$ implies that every solution exists globally. 
The above results show that $1 / 2$ is the critical number which criticize the existence and nonexistence of global solutions.

Remark 2. Considering the general equations

$$
\begin{cases}(a(u))_{t}=\Delta u & \text { in } \Omega \times(0, T), \\ \frac{\partial u}{\partial n}=b(u) & \text { on } \partial \Omega \times(0, T), \\ u(x, 0)=u_{0}(x) & \text { in } \Omega,\end{cases}
$$

where $\Omega \subset \mathbb{R}^{N}$ is a bounded domain with smooth boundary $\partial \Omega, u_{0} \in L^{\infty}(\Omega)$ and $u_{0}(x) \geq \delta$ for some small $\delta>0$. $a(s), b(s) \in C^{1}(0,+\infty), a(s), b(s)$ and $a^{\prime}(s)$ are positive functions for $s>0$. Combining the proof of Theorem 1 with the methods of [9] and [12], we can prove the following results:

Assume that there exist some $m \geq 0$ and $M>0$, such that $\left(m b^{\prime}(s)+1\right) / a^{\prime}(s)$ is monotone (nonincreasing or nondecreasing) for $s \geq M$.

(I). If

$$
\int^{+\infty} \frac{a^{\prime}(s)}{b(s)\left(m b^{\prime}(s)+1\right)} d s<+\infty
$$

then every solution blows up in finite time.

(II). If

$$
\int^{+\infty} \frac{d s}{b(s)}=+\infty \text { and } \int^{+\infty} \frac{a^{\prime}(s)}{b(s)\left(m b^{\prime}(s)+1\right)} d s=+\infty
$$

then every solution exists globally.

However, if

$$
\int^{+\infty} \frac{d s}{b(s)}<+\infty \text { and } \int^{+\infty} \frac{a^{\prime}(s)}{b(s)\left(m b^{\prime}(s)+1\right)}=+\infty,
$$

then the situations are very complicated. Theorem 1 and Remark 1 show that, for some special functions $a(s)$ and $b(s)$, every solution also exists globally. But, if we choose

$$
b(s)=\exp \left(e^{s}\right), \quad a(s)=\int_{0}^{s} \frac{b(y) b^{\prime}(y)}{1+y} d y,
$$

we have not obtained any results on the existence and nonexistence of positive global solutions.

\section{REFERENCES}

1. N. Wolanski, Global behavior of positive solutions to nonlinear diffusion problems with nonlinear absorption through the boundary, SIAM J. Math. Anal., 24(2)(1993), 317-326. MR 35023

2. D.G. Aronson, Regularity properties of flows through porous media, SIAM J. Appl. Math., 17(1969), 827-868. MR 41:656

3. D.G. Aronson, Regularity properties of flows through porous media: A counterexample, SIAM J. Appl. Math., 19(1970), 299-307. MR 42:683

4. J. G. Berryman and C.J. Holland, Stability of the separable solution for fast diffusion, Arch. Rational Mech. Anal., 74(1980), 279-288. MR 81m:35065

5. H. Levine and L.E. Payne, Nonexistence theorems for the heat equation with nonlinear boundary condition and for the porous medium equation backward in time, J. of Diff. Eqns., 16(1974), 319-334. MR 57:10235

6. J.R. Anderson, Local existence and uniqueness of solutions of degenerate parabolic equations, Commu. In Partial Diff. Eqns., 16(1)(1991), 105-143. MR 92d:35163 
7. W. Walter, On existence and nonexistence in the large of solutions of parabolic differential equation with a nonlinear boundary condition, SIAM J. Math. Anal., 6(1)(1975), 85-90. MR 51:1122

8. J. López Gómez, V. Márquez and N. Wolanski, Blow up results and localization of blow up points for the heat equation with a nonlinear boundary condition, J. of Diff. Eqns., 92(1991), 384-401. MR 92j:35098

9. M.X. Wang and Y.H. Wu, Global existence and blow up problems for quasilinear parabolic equations with nonlinear boundary conditions, SIAM J. Math. Anal., 24(6)(1993), 1515-1521. MR 95c:35132

10. J. Filo, Diffusivity versus absorption through the boundary, J. of Diff. Eqns., 99(1992), 281305. MR 94d:35083

11. Y.H. Wu, Remarks on the diffusivity versus absorption through the boundary, submitted to J. Sys. Sci. \& Math. Scis., in Chinese.

12. M.X. Wang, Long time behaviors of solutions of a quasilinear parabolic equation with nonlinear boundary condition, Acta Math. Sinica 39 (1) (1996), 118-124, in Chinese.

13. M. Chipot, F. Fila and P. Quittner, Stationary solutions, blow up and convergence to stationary solutions for semilinear parabolic equations with nonlinear boundary conditions, Acta Math. Univ. Comenian, 60(1991), 35-103. MR 92h:35110

14. H. Levine, L. Payne, P. Sacks and B. Straughan, Analysis of a convective reaction-diffusion equation (II), SIAM J. Math. Anal., 20(1989), 133-147. MR 90e:35092

15. Y.H.Wu, M.X.Wang, Existence and nonexistence of global solution of nonlinear parabolic equation with nonlinear boundary condition, Chinese Ann. of Math.,13(1995), ser. B, 371378. CMP 96:07

Department of Mathematics and Mechanics, Southeast University, Nanjing 210018, P.R. CHINA

E-mail address: mxwang@seu.edu.cn

Institute of Applied Physics and Computational Mathematics, Beijing 100088, P.R. CHINA 